Article

\title{
Morphological Characterization of Deciduous Enamel and Dentin in Patients Affected by Osteogenesis Imperfecta
}

\author{
Uros Josic $^{1}$, Tatjana Maravic ${ }^{1}$, Maurizio Bossù ${ }^{2}$, Milena Cadenaro ${ }^{3,4}\left({ }^{\circledR}\right.$, Allegra Comba $^{1}$, \\ Gaetano Ierardo ${ }^{2}$, Antonella Polimeni ${ }^{2}\left(\mathbb{D}\right.$, Federica Florenzano ${ }^{1}$, Lorenzo Breschi ${ }^{1}(\mathbb{C})$ \\ and Annalisa Mazzoni ${ }^{1, *}$ \\ 1 Department of Biomedical and Neuromotor Sciences, DIBINEM, University of Bologna-Alma Mater \\ Studiorum, 40125 Bologna, Italy; uros.josic2@unibo.it (U.J.); tatjana.maravic@studio.unibo.it (T.M.); \\ allegra.comba@unibo.it (A.C.); federica.florenzano3@unibo.it (F.F.); lorenzo.breschi@unibo.it (L.B.) \\ Department of Oral and Maxillofacial Science, "Sapienza" University of Rome, 00185 Rome, Italy; \\ maurizio.bossu@uniroma1.it (M.B.); gaetano.ierardo@uniroma1.it (G.I.); \\ antonella.polimeni@uniroma1.it (A.P.) \\ 3 Department of Medical Sciences, University of Trieste, 34149 Trieste, Italy; mcadenaro@units.it \\ 4 Institute for Maternal and Child Health - IRCCS “Burlo Garofolo", 34137 Trieste, Italy \\ * Correspondence: annalisa.mazzoni@unibo.it
}

Received: 26 September 2020; Accepted: 3 November 2020; Published: 5 November 2020

\begin{abstract}
The purpose of this study was to clarify the structural and ultrastructural alterations of the enamel and dentin collagen network in the deciduous teeth of children affected by osteogenesis imperfecta (OI) using field-emission in-lens scanning electron microscopy (FEI-SEM) and transmission electron microscopy (TEM) analyses. Exfoliated primary teeth were collected from children with a diagnosis of $\mathrm{OI}$ and from healthy individuals $(N=24)$. Tooth slices containing both dentin and enamel were fixed, dehydrated and dried, gold sputtered, and observed using FEI-SEM. Additional dentin fragments were decalcified, dehydrated, embedded in resin, cut, and processed for TEM analysis. Under FEI-SEM, the enamel in OI-affected children showed an irregular prism distribution with the enamel hydroxyapatite crystals unpacked. Ultrastructural correlative analysis of the dentin in patients affected by OI showed an altered collagen pattern with a low density. In some areas, teeth in OI patients showed a reduction in the number of dentinal tubules, with odontoblastic process missing in most of the tubules. The presence of altered dentine and enamel organization in OI children was firmly established at an ultrastructural level, but additional biochemical studies are necessary in order to clarify quantitatively and qualitatively the collagenic and non-collagenic proteins in this disorder.
\end{abstract}

Keywords: deciduous teeth; dentinogenesis imperfecta; osteogenesis imperfecta; enamel; dentin; ultrastructural diagnosis

\section{Introduction}

Type I collagen is the most abundant collagen of the human body and constitutes $85-90 \%$ of the dentin organic matrix. It possesses a triple helix containing two alpha 1 and one alpha 2 polypeptides, which are expressed from COL1A1 and COL1A2 [1]. Mutations in these genes cause osteogenesis imperfecta (OI), an autosomal genetic disorder that affects connective tissues and, more specifically, the proteins involved in the organization of the extracellular matrix. The main symptoms of this disease are attributable to fragile bones, blue sclerae, hearing loss, scoliosis, and dentinogenesis imperfecta (DGI) [2]. OI is usually classified on the basis of clinical and radiologic criteria into four types, each 
further subdivided based on the presence or absence of the developmental dental defect known as DGI [3].

DGI is a hereditary dentin disorder characterized by abnormal dentin structure affecting either the primary or both the primary and secondary dentition. DGI is reported to have an incidence of 1 in 6000 to 1 in 8000 and is inherited in a simple autosomal dominant mode, with high penetrance and a low mutation rate [4]. To date, various authors have sought to recommend adequate nomenclature for DGI, but confusion still exists among clinicians dealing with this population of patients [5]. Major advances have been made in the field of molecular genetics [6], and new classification proposals for dentinal defects have been made based on the knowledge of molecular pathophysiology [7]. However, the most commonly used classification still remains the one proposed by Shields, and overall, DGI associated with OI can be regarded as a syndromic form of OI or DGI type I [8]. All other forms of DGI appear to result from mutations in the gene encoding dentin sialophosphoprotein (DSPP), suggesting that these conditions are allelic [9]. Table 1 shows a synthesis of proposed classifications for DGI disorders, including aetiology and clinical manifestations.

Table 1. Overview of classification for DGI disorders including aetiology and clinical manifestations. DGI—dentinogenesis imperfecta; OI—osteogenesis imperfecta; DSPP—dentin sialophosphoprotein.

\begin{tabular}{ccc}
\hline Classification & Aetiology & Clinical Manifestations \\
\hline DGI type I (Shields) or & Mutation in COL1A1 and & $\begin{array}{c}\text { Amber and translucent teeth with attrition in } \\
\text { pGimary and secondary dentition }\end{array}$ \\
$\begin{array}{c}\text { DGI type II (Shields) or } \\
\text { moderate form }\end{array}$ & COL1A2 gene & Mutation in DSPP gene \\
$\begin{array}{c}\text { DGI type III (Shields) or } \\
\text { severe form }\end{array}$ & Mutation in DSPP gene & Variable clinical features, resembling those in DGI \\
type I and II
\end{tabular}

Regardless of the classification used, the clinical features of patients with OI-associated DGI remain characteristic and include brownish teeth discoloration, crown fractures, dentin attrition, and late dentition [10]. A previous study demonstrated that there is not always a direct correlation between OI and DGI. In other words, it was found that $62.5 \%$ of children with OI displayed clinically visible dental alterations [11]. Overall, it is considered that the prevalence of DGI in OI patients ranges from $8 \%$ up to $100 \%$ [12]. The primary dentition is usually more severely affected than permanent teeth [13], and, alongside the patients' young age, this further complicates treatment procedures and their outcomes. Therefore, it is of importance to understand the structure of the enamel and dentin at the microscopic level in order to provide adequate oral health care for patients suffering from OI. Previous studies $[11,14,15]$ have reported pathologically altered dentin and essentially normal enamel in patients suffering from OI. However, some authors [16] did report slight changes in the structure of the enamel in DGI teeth, and these findings may awake suspicion that even the enamel in OI patients may be affected. Thus, the aim of the present study was to clarify the structural and ultrastructural alterations of enamel in the deciduous teeth of patients affected by OI by means of field-emission in-lens scanning electron microscopy (FEI-SEM) analysis and to evaluate the dentin collagen network organization by means of correlative FEI-SEM and TEM analyses. The null hypotheses of the present study were that there are no ultrastructural alterations in the (1) enamel or (2) dentin of the deciduous teeth of OI patients, when compared to deciduous teeth from healthy children.

\section{Materials and Methods}

All reagents were purchased from Sigma Aldrich (St. Louis, MO, USA), unless otherwise specified. Exfoliated primary teeth $(N=24)$ were used in this study: 12 teeth were collected from patients affected by OI, while an additional 12 primary teeth were collected from healthy patients and used as controls. The diagnosis of OI had been previously confirmed by obtaining the data from patients' medical charts. The age range of donors was 6-12 years. Teeth selected for the study were free from 
caries and fillings. The consent form and experimental protocol was approved by the Department of Oral and Maxillofacial Science, "Sapienza" University of Rome, Rome, Italy.

Collected teeth were then processed for morphological examination under field-emission in-lens scanning electron microscopy (FEI-SEM) and transmission electron microscopy (TEM) analyses.

\subsection{Tissue Processing for FEI-SEM Analysis}

After washing with phosphate buffer, each tooth was cut perpendicular to the long axis to produce 1-mm-thick enamel-dentin disks using a slow speed diamond saw (Remet, Bologna, Italy) under intense and continuous water-cooling. Specimens were then fixed with $0.01 \%$ glutaraldehyde and $4 \%$ paraformaldehyde in $0.1 \mathrm{M}$ phosphate buffer for $2 \mathrm{~h}$ and rinsed with $0.1 \mathrm{M}$ phosphate buffer. After dehydration in an ascending ethanol series $(30 \%, 50 \%, 70 \%, 90 \%, 95 \%, 100 \%)$, specimens were critical point dried, mounted on stubs using a conductive tape, and gold sputtered using a Balzers Med 010 Multicoating Systemgold (Bal-Tec AG, Liechtenstein). Observations were performed via FEI-SEM (JSM 890, JEOL, Tokyo, Japan).

\subsection{Tissue Processing for TEM Analysis}

For TEM analysis, teeth were fixed with $0.1 \%$ glutaraldehyde and $4 \%$ paraformaldehyde in PBS for $2 \mathrm{~h}$ at room temperature, rinsed with PBS, and demineralized in aqueous ethylenediaminetetraacetic acid (EDTA) (0.5 M, pH 7.0) for up to 3 months. Samples were dehydrated in graded concentrations of ethanol and embedded in LR White resin (London Resin, Berkshire, UK). Semithin sections (1 $\mu \mathrm{m})$ were cut with glass knives on a Reichert Jung Ultracut E ultramicrotome and stained with toluidine blue. Selected areas of the 1- $\mu \mathrm{m}$-thick sections were trimmed for ultrathin sectioning, and 80-nm-thick sections were prepared and mounted on formvar carbon-coated nickel grids and observed using a Zeiss EM 109 electron microscope (Zeiss, Oberkochen, Germany).

\section{Results}

At the macroscopic level, all 12 teeth collected from OI patients showed abnormal, yellow/brownish or opalescent grey discolorations. Teeth from healthy donors showed no signs of pathological changes at the macroscopic level.

The structural and ultrastructural features of the enamel and dentin in sound primary teeth and teeth from patients affected by OI were assayed. Two investigators independently observed and described the images. They had no previous knowledge of whether the observed samples were harvested from healthy or OI donors.

In teeth from healthy donors, SEM analysis presented enamel with rare micropores and abundant prisms with an undulated and sinuous trend (Figure 1C,D). Enamel from patients with OI, on the contrary, showed an irregular trend of the enamel prisms (Figure 2C) and the crystals of hydroxyapatite were found to be unpacked (Figure 2D) in all teeth subjected to observation.

In the dentin, SEM examination of the surface confirmed the presence of compact collagen fibers (Figure 1B). The intertubular tissue was observed to be stably packed (Figure 1A), while in pathological dentin, it had lower surface density compared with the control (Figure 2A,B). 

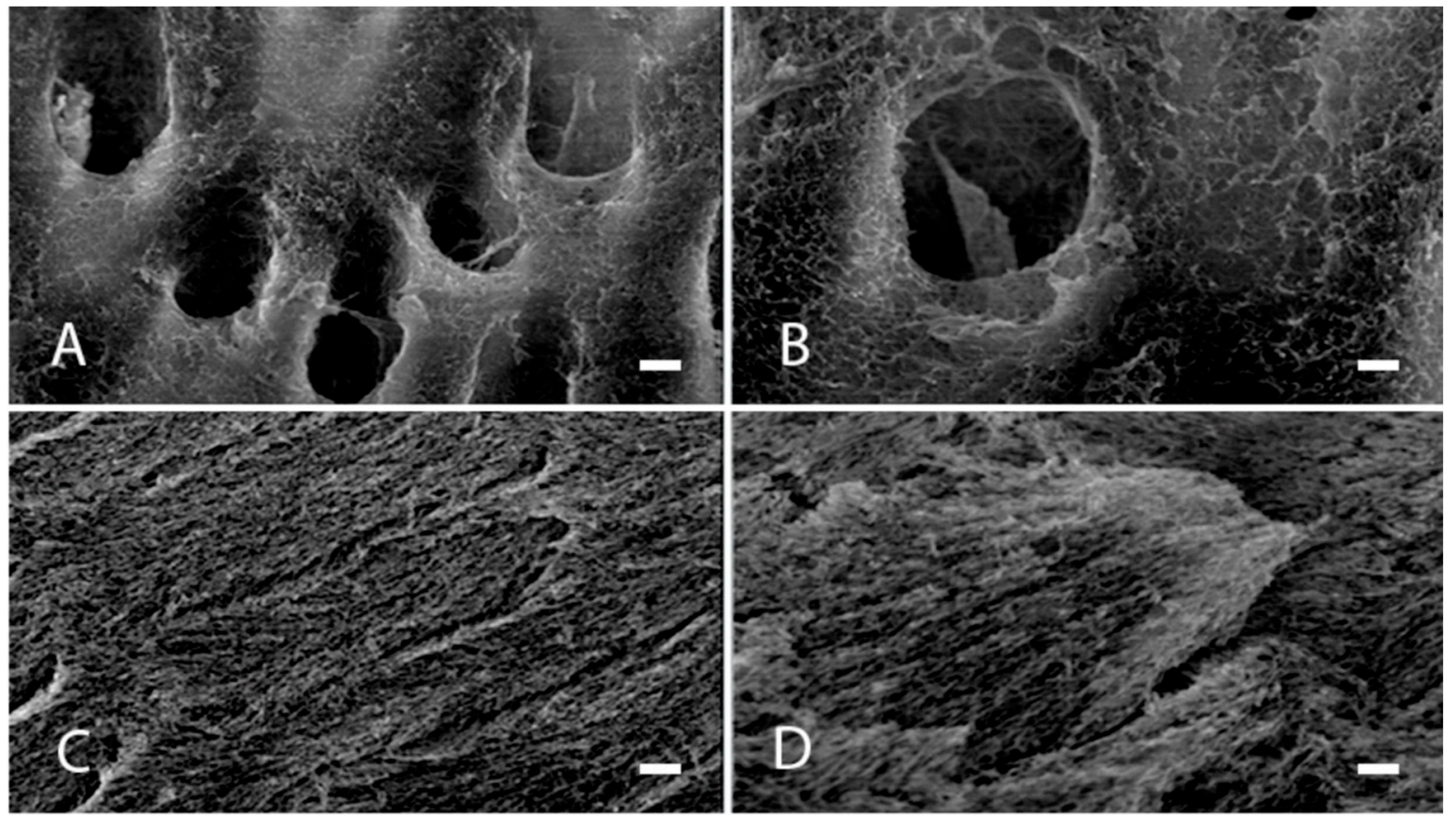

Figure 1. SEM analysis of enamel and dentin from healthy donors. (A): Intertubular tissue; (B): Collagen fibers; (C,D): Enamel with rare micropores and abundant prisms.
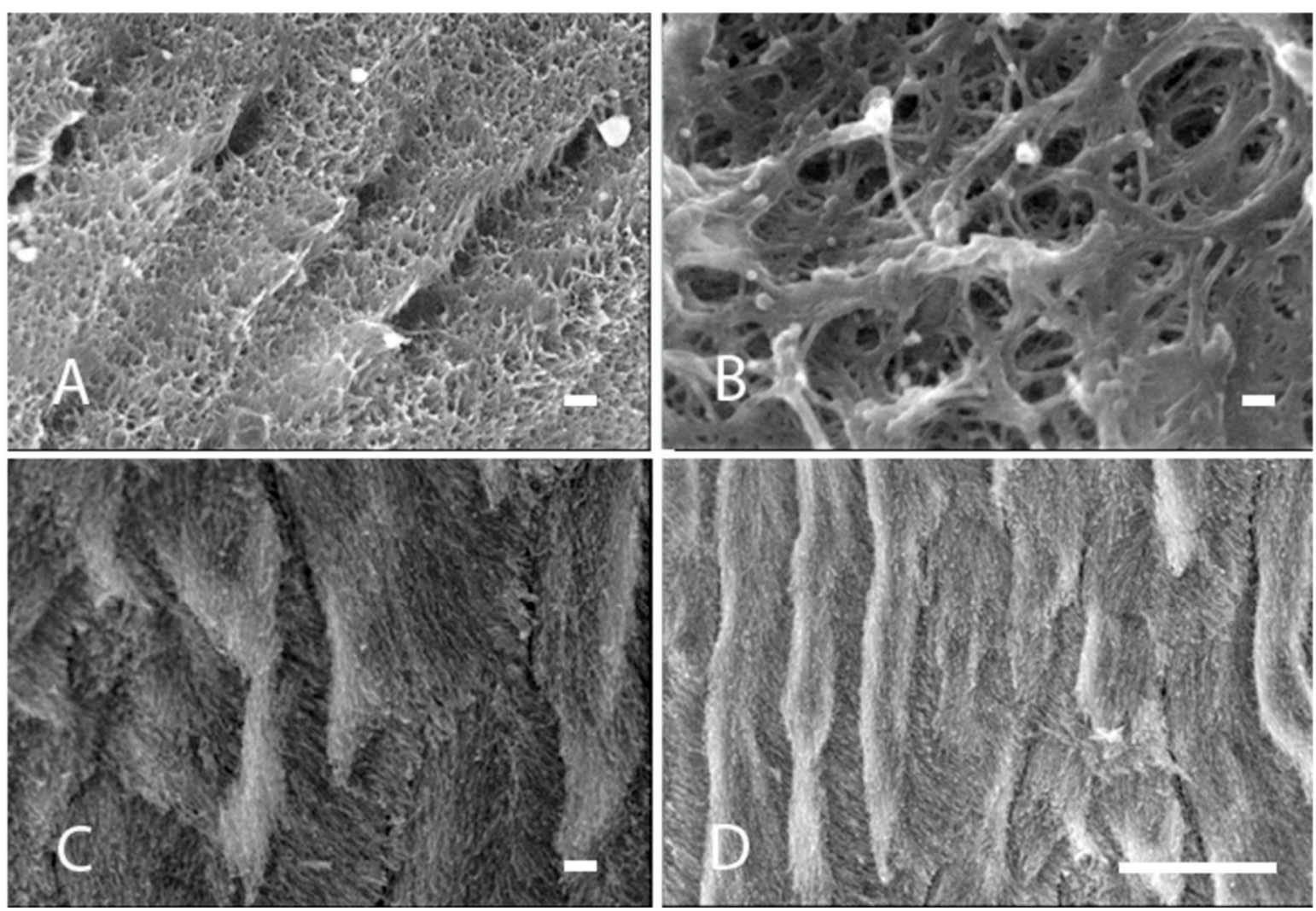

Figure 2. SEM analysis of enamel and dentin from OI patients. (A,B): Intertubular tissue and collagen fibers; (C): Enamel prisms; (D): Unpacked crystals of hydroxyapatite.

Morphological studies showed clear differences between normal dentin and dentin found in OI patients. Semithin section analysis of the normal dentin displayed a regular organization of tubules (Figure 3A), whereas dentin in OI patients showed dysplastic and irregular dentinal tubules 
(Figure 3B,C). In addition, the dentin in OI children presented tubules without odontoblastic process, but with large collagen fibers that entirely cross the tubule (Figure $3 \mathrm{C}$ ). This particular characteristic was mainly evident in TEM analysis (Figure 4D,E).
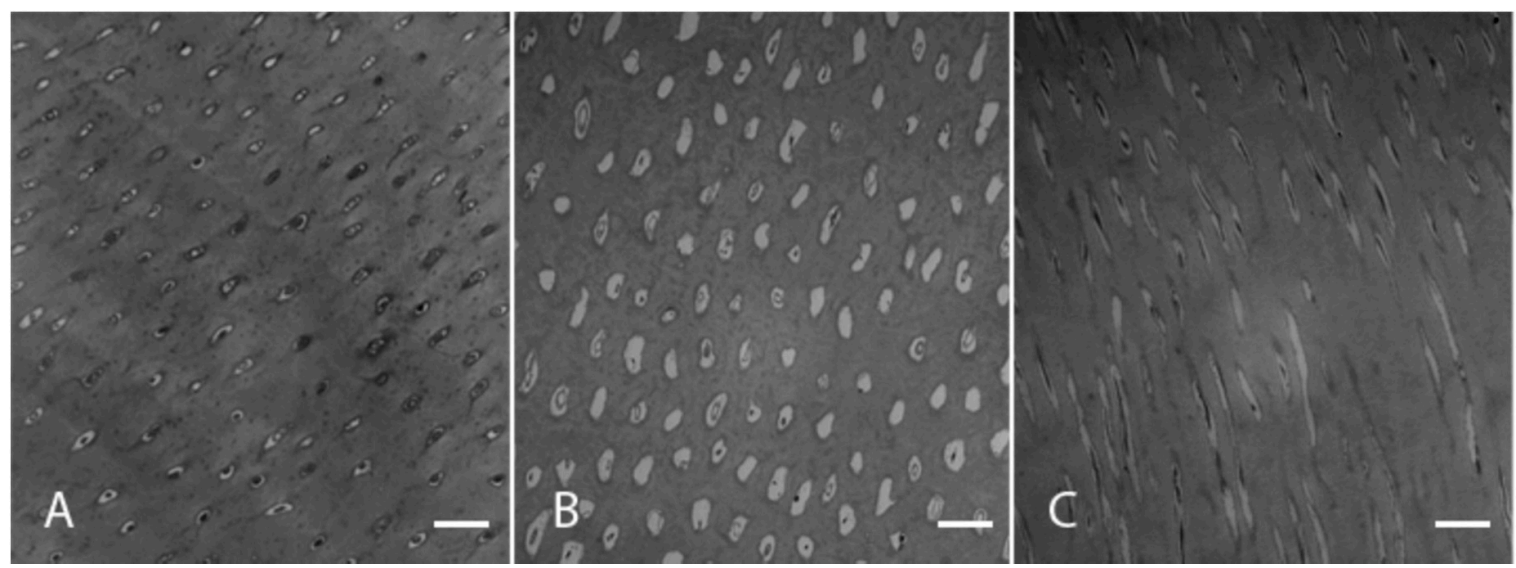

Figure 3. Morphological analysis of dentin from healthy and OI donors. (A): Dentin from healthy donors; (B,C): Dentin from OI donors without odontoblastic process.

In dentin from healthy donors, TEM analysis showed normal morphological features as well as compacted collagen with a fibrillar scaffold and regular odontoblastic process residues. One was embedded in a dense network of collagen fibers highlighted by contrast of the grids with tannic acid (Figure $4 \mathrm{~A}-\mathrm{C}$ ).
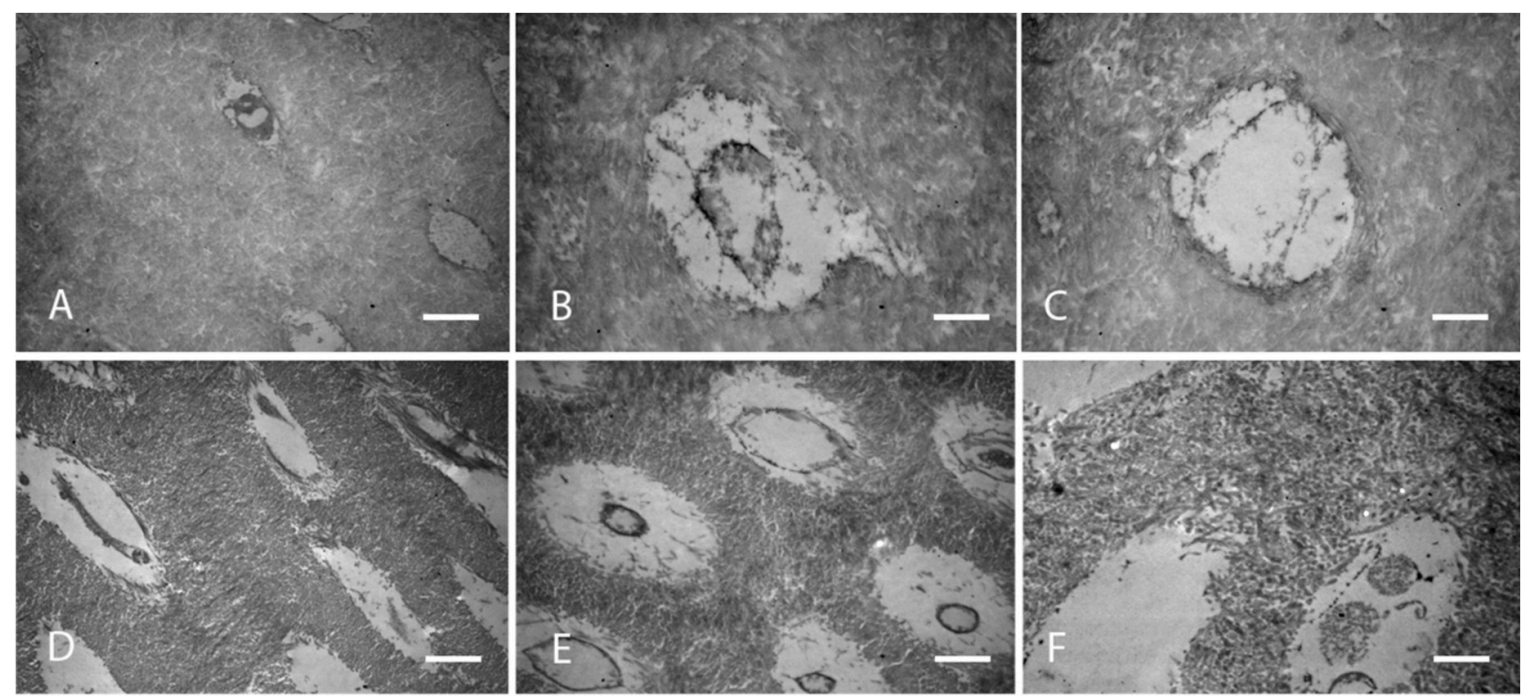

Figure 4. TEM analysis of dentin from healthy and OI donors. (A-C): Compacted collagen with a fibrillar scaffold and regular odontoblastic process in healthy donors; (D): Absence of odontoblastic process in dentin tubules in OI patients; $(\mathbf{E}, \mathbf{F})$ : Unusual collagen circles with unorganized and unpacked collagen in OI patients.

On the contrary, dentin from patients affected by OI showed areas lacking dentin tubules; in most tubules, odontoblastic processes were absent (Figure 4D), and the tubular area presented collagen bundles and unusual and characteristic collagen circles (Figure 4E,F). The dentinal tissue was observed to be of low density; in fact, collagen was lax, unorganized, and unpacked (Figure 4F). Large interfibrillar free spaces were evident (Figure 4F). In dentin tubules, some necrotic aspects could also be identified 
(Figure 4F). Overall, the above described changes related to dentin were seen in all teeth collected from OI patients.

\section{Discussion}

This morphological approach to the study of normal and pathological dentin demonstrates the effectiveness of correlative FEI-SEM/TEM analysis. We have established, at an ultrastructural level, the presence of altered dentin and enamel organization in OI patients. Hence, both null hypotheses can be rejected.

Dentinogenesis is a highly regulated developmental process that occurs both prenatally and postnatally, during which a layer of predentin is always present between the odontoblasts and the mineralized front [17]. As odontoblasts migrate towards the pulp, they leave behind an extension referred to as the odontoblastic process, which then penetrates the predentin. At this stage, predentin is not mineralized and is composed of a collagen network, which gradually becomes mineralized as the extension of the odontoblastic process results in protein secretion necessary for the mineralization process [18]. With mutations in the genes encoding collagen, dentin defects may occur [13]. The primary dentition is usually more severely affected than permanent teeth, which can be explained by the faster formation of the primary teeth and the fact they are formed in the fetal period during which high collagen expression is present, while the permanent teeth are formed entirely after birth [19]. In some cases, dental changes are the most visible clinical manifestation of OI, and early diagnosis and understanding of the ultrastructural changes found in these teeth are essential for maintaining or improving the oral health of OI patients and their quality of life [20].

The present study demonstrated irregular enamel prisms and unpacked hydroxyapatite crystals. Similar to our findings, Lindau et al. reported more irregularly mineralized enamel in patients with OI. An almost nonprismatic layer was observed within the superficial region of the enamel, and more irregular arrangement of the prism pattern was present close to the dentin-enamel junction [16]. Contrary to our study results, Lygidarkis et al. reported no difference between OI-affected and healthy enamel [15]. In a more recent study by Majorana et al., optical microscopy and confocal laser-scanning microscopy (CLSM) showed normal enamel with regular prismatic structure in OI patients [11]. One possible explanation of the different findings in our study may lie in the facts that children who are more severely affected by OI often have a more severe dental phenotype and that enamel malformations can result from alterations in a gene that is not known or considered to have a role in enamel formation [12,21]. The range of enamel malformations observed in our SEM analysis is probably compatible with enamel hypomaturation, which may explain the susceptibility of teeth affected by OI-related DGI to severe attrition, or even fractures.

The histological evaluation of dentin in patients affected with OI has been of interest, and many methods have been employed in investigating its structure $[11,15,22,23]$. Regardless of the type of OI (I, III, or IV), distinct pathological changes in dentin have been observed and reported in the literature [11]. The results of our study are in agreement with previous findings and support the observations of irregular dentinal tubules and areas lacking dentin tubules. In previous studies, a reduction in the number of dentinal tubules and the presence of narrow-diameter and short tubules which did not penetrate through the whole thickness of the dentine were reported [15]. Aberrant dentin with randomly oriented collagen fibers with areas of occluded tubules was also seen using CLSM [11]. The level of ultrastructural dentinal changes observed in our study is likely due to collagen defects in patients with OI and the dysfunction of odontoblasts [14]. Interestingly, the combined FEI-SEM/TEM approach used in this study allowed us to notice areas of necrosis in some of the dentinal tubules, which, to the best of our knowledge, has not been reported before. We speculate that a possible cause of the necrotic areas is premature death or dedifferentiation of the odontoblasts and the consequent disappearance of the cytoplasmic process [19].

When treating children with OI, attention should be given to preventing enamel attrition and rapid wear of the poorly mineralized dentin, as well as preventing caries and periodontal disease. 
The American Academy for Paediatric Dentistry guideline recommends that children affected with DGI should undergo intensive preventive interventions, among which fluoride application and desensitizing agents that reduce tooth sensitivity have been shown to be effective [24]. Restorative care is frequently needed and should be carried out prior to excessive tooth wear and the loss of vertical dimension. For severe cases of type II DGI, Sapir et al. suggested that primary incisors and canines should be restored with composite restorations, while molars should be protected with preformed crowns [25]. However, in light of the observations of our study, it should be considered that the adhesion of composite restorations placed on primary teeth in OI patients may be compromised due to the altered structure of collagen fibers. When placing composite restorations, the mineral component of the superficial layer of dentin is removed by acid etching in etch-and-rinse adhesives, or with acidic primers in self-etch adhesives. This procedure leaves behind an exposed collagen matrix, which is then infiltrated with adhesive resin monomers, leading to the creation of a hybrid layer-a structure whose integrity is essential for the durability of the restorations. Even in favorable clinical situations where a sound collagen structure is present, adhesive monomers are not able to entirely infiltrate the exposed collagen matrix, thus leaving unprotected collagen fibrils at the bottom of the hybrid layer [1]. Degradation of the hybrid layer is a result of the presence of endogenous enzymes and the consequent hydrolysis of collagen fibers which are not completely embedded in polymerized adhesive resins. Hydrolytic processes contribute to the weakening of the adhesive joint between the resin material and dentin and are responsible for the loss of bond strength over time [26-28]. Due to the presence of altered collagen fibrils, adhesive procedures applied on the teeth in OI-affected children could result in the formation of a weaker hybrid layer. The laxed and unorganized collagen fibrils which are infiltrated by resin monomers within the hybrid layer may be more susceptible to micromechanical disruption during occlusal loads. This mechanical aspect of hybrid layer degradation, combined with the above described hydrolytic processes and their possible impact on the clinical performance of composite restorations, should be kept in mind while attending to OI patients. Moreover, the use of cross-linkers-molecules that have the ability to create chemical bonds between the collagen molecules and thereby render collagen more mechanically resistant-should be considered in patients with OI. These molecules have been widely investigated in healthy permanent teeth $[29,30]$, and their efficacy in structurally altered OI-affected teeth should be studied further.

Finally, the potential limitations of the study should be taken into consideration. In this research, no quantitative data were used and, consequently, no statistical analysis was performed due to the nature of the study. Instead, a descriptive approach was used when analyzing representative images of teeth from both healthy and OI donors, similarly to previously published studies $[11,15,16]$. In order to eliminate the risk of bias, two experienced researchers observed and described the images independently from each other, after which they discussed them together. Even though no statistical analysis was performed, this type of qualitative analysis allowed us to establish fine distinctions on the ultrastructural level between teeth from healthy and OI children.

\section{Conclusions}

The results of this study clearly indicate an ultrastructural alteration in both the enamel and dentin in the teeth of children affected by OI that probably underlies the clinical manifestations and treatment difficulties in this group of patients. Additional biochemical studies are necessary in order to quantitatively and qualitatively define the collagenic and non-collagenic proteins in teeth associated with OI.

Author Contributions: Conceptualization, A.M., M.C. and L.B.; methodology, U.J., T.M., F.F.; investigation, M.B., G.I. and A.P.; resources, M.B., G.I. and A.P.; writing-original draft preparation, U.J., T.M. and A.C.; writing-review and editing, L.B., A.M.; visualization, U.J., F.F. and A.C.; supervision, A.M. and M.C.; All authors have read and agreed to the published version of the manuscript.

Funding: This research received no external funding. 
Acknowledgments: The authors wish to thank Aurelio Valmori (University of Bologna) for photographical assistance and Rosa Curci for laboratory processing.

Conflicts of Interest: The authors declare no conflict of interest.

\section{References}

1. Breschi, L.; Maravić, T.; Cunha, S.R.; Comba, A.; Cadenaro, M.; Tjäderhane, L.; Pashley, D.H.; Tay, F.R.; Mazzoni, A. Dentin bonding systems: From dentin collagen structure to bond preservation and clinical applications. Dent. Mater. 2018, 34, 78-96. [CrossRef]

2. Burnei, G.; Vlad, C.; Georgescu, I.; Gavriliu, T.S.; Dan, D. Osteogenesis imperfecta: Diagnosis and treatment. J. Am. Acad. Orthop. Surg. 2008, 16, 356-366. [CrossRef] [PubMed]

3. Thomas, I.H.; DiMeglio, L.A. Advances in the Classification and Treatment of Osteogenesis Imperfecta. Curr. Osteoporos. Rep. 2016, 14, 1-9. [CrossRef] [PubMed]

4. Kim, J.W.; Simmer, J.P. Hereditary dentin defects. J. Dent. Res. 2007, 86, 392-399. [CrossRef] [PubMed]

5. Chetty, M.; Roberts, T.; Stephen, L.X.; Beighton, P. Hereditary dentine dysplasias: Terminology in the context of osteogenesis imperfecta. Br. Dent. J. 2016, 221, 727-730. [CrossRef] [PubMed]

6. Maciejewska, I.; Chomik, E. Hereditary dentine diseases resulting from mutations in DSPP gene. J. Dent. 2012, 40, 542-548. [CrossRef] [PubMed]

7. de La Dure-Molla, M.; Philippe Fournier, B.; Berdal, A. Isolated dentinogenesis imperfecta and dentin dysplasia: Revision of the classification. Eur. J. Hum. Genet. 2015, 23, 445-451. [CrossRef]

8. Shields, E.D.; Bixler, D.; El-Kafrawy, A.M. A proposed classification for heritable human dentine defects with a description of a new entity. Arch. Oral Biol. 1973, 18, 543-553. [CrossRef]

9. Hart, P.S.; Hart, T.C. Disorders of human dentin. Cells Tissues Organs 2007, 186, 70-77. [CrossRef] [PubMed]

10. Barron, M.J.; McDonnell, S.T.; Mackie, I.; Dixon, M.J. Hereditary dentine disorders: Dentinogenesis imperfecta and dentine dysplasia. Orphanet J. Rare Dis. 2008, 3, 31. [CrossRef]

11. Majorana, A.; Bardellini, E.; Brunelli, P.C.; Lacaita, M.; Cazzolla, A.P.; Favia, G. Dentinogenesis imperfecta in children with osteogenesis imperfecta: A clinical and ultrastructural study. Int. J. Paediatr. Dent. 2010, 20, 112-118. [CrossRef]

12. Andersson, K.; Dahllöf, G.; Lindahl, K.; Kindmark, A.; Grigelioniene, G.; Åström, E.; Malmgren, B. Mutations in COL1A1 and COL1A2 and dental aberrations in children and adolescents with osteogenesis imperfecta-A retrospective cohort study. PLoS ONE 2017, 12, e0176466. [CrossRef] [PubMed]

13. Vital, S.O.; Gaucher, C.; Bardet, C.; Rowe, P.; George, A.; Linglart, A.; Chaussain, C. Tooth dentin defects reflect genetic disorders affecting bone mineralization. Bone 2012, 50, 989-997. [CrossRef] [PubMed]

14. Hall, R.K.; Maniere, M.C.; Palamara, J.; Hemmerle, J. Odontoblast dysfunction in osteogenesis imperfecta: An LM, SEM, and ultrastructural study. Connect. Tissue Res. 2002, 43, 401-405. [CrossRef]

15. Lygidakis, N.A.; Smith, R.; Oulis, C.J. Scanning electron microscopy of teeth in osteogenesis imperfecta type I. Oral Surg. Oral Med. Oral Pathol. Oral Radiol. Endod. 1996, 81, 567-572. [CrossRef]

16. Lindau, B.M.; Dietz, W.; Hoyer, I.; Lundgren, T.; Storhaug, K.; Noren, J.G. Morphology of dental enamel and dentine-enamel junction in osteogenesis imperfecta. Int. J. Paediatr. Dent. 1999, 9, 13-21. [CrossRef]

17. Arana-Chavez, V.E.; Massa, L.F. Odontoblasts: The cells forming and maintaining dentine. Int. J. Biochem. Cell Biol. 2004, 36, 1367-1373. [CrossRef]

18. Nino-Barrera, J.L.; Gutierrez, M.L.; Garzon-Alvarado, D.A. A theoretical model of dentinogenesis: Dentin and dentinal tubule formation. Comput. Methods Programs Biomed. 2013, 112, 219-227. [CrossRef]

19. Luder, H.U.; van Waes, H.; Raghunath, M.; Steinmann, B. Mild dental findings associated with severe osteogenesis imperfecta due to a point mutation in the alpha 2(I) collagen gene demonstrate different expression of the genetic defect in bone and teeth. J. Craniofacial Genet. Dev. Biol. 1996, 16, 156-163.

20. Teixeira, C.S.; Santos Felippe, M.C.; Tadeu Felippe, W.; Silva-Sousa, Y.T.; Sousa-Neto, M.D. The role of dentists in diagnosing osteogenesis imperfecta in patients with dentinogenesis imperfecta. J. Am. Dent. Assoc. 2008, 139, 906-914. [CrossRef]

21. Santos, M.C.; Line, S.R. The genetics of amelogenesis imperfecta: A review of the literature. J. Appl. Oral Sci. 2005, 13, 212-217. [CrossRef] 
22. Lindau, B.; Dietz, W.; Lundgren, T.; Storhaug, K.; Noren, J.G. Discrimination of morphological findings in dentine from osteogenesis imperfecta patients using combinations of polarized light microscopy, microradiography and scanning electron microscopy. Int. J. Paediatr. Dent. 1999, 9, 253-261. [CrossRef] [PubMed]

23. Orsini, G.; Majorana, A.; Mazzoni, A.; Putignano, A.; Falconi, M.; Polimeni, A.; Breschi, L. Immunocytochemical detection of dentin matrix proteins in primary teeth from patients with dentinogenesis imperfecta associated with osteogenesis imperfecta. Eur. J. Histochem. 2014, 58, 2405. [CrossRef] [PubMed]

24. American Academy of Pediatric Dentistry. Guideline on dental management of heritable dental developmental anomalies. Pediatr. Dent. 2013, 35, E179-E184.

25. Sapir, S.; Shapira, J. Dentinogenesis imperfecta: An early treatment strategy. Pediatr. Dent. 2001, 23, $232-237$. [PubMed]

26. Breschi, L.; Mazzoni, A.; Ruggeri, A.; Cadenaro, M.; Di Lenarda, R.; De Stefano Dorigo, E. Dental adhesion review: Aging and stability of the bonded interface. Dent. Mater. 2008, 24, 90-101. [CrossRef]

27. Frassetto, A.; Breschi, L.; Turco, G.; Marchesi, G.; Di Lenarda, R.; Tay, F.R.; Pashley, D.H.; Cadenaro, M. Mechanisms of degradation of the hybrid layer in adhesive dentistry and therapeutic agents to improve bond durability-A literature review. Dent. Mater. 2016, 32, e41-e53. [CrossRef]

28. Tjäderhane, L.; Nascimento, F.D.; Breschi, L.; Mazzoni, A.; Tersariol, I.L.; Geraldeli, S.; Tezvergil-Mutluay, A.; Carrilho, M.; Carvalho, R.M.; Tay, F.R.; et al. Strategies to prevent hydrolytic degradation of the hybrid layer-A review. Dent. Mater. 2013, 29, 999-1011. [CrossRef]

29. Mazzoni, A.; Angeloni, V.; Comba, A.; Maravić, T.; Cadenaro, M.; Tezvergil, A.; Pashley, D.H.; Tay, F.R.; Breschi, L. Cross-linking effect on dentin bond strength and MMPs activity. Dent. Mater. 2018, 34, $288-295$. [CrossRef]

30. Scheffel, D.L.; Hebling, J.; Scheffel, R.H.; Agee, K.A.; Ca, D.S.C.; Turco, G.; Breschi, L.; Mazzoni, A.; Costa, C.A.D.S.; Pashley, D.H. Stabilization of dentin matrix after cross-linking treatments, in vitro. Dent. Mater. 2014, 30, 227-233. [CrossRef]

Publisher's Note: MDPI stays neutral with regard to jurisdictional claims in published maps and institutional affiliations.

(C) 2020 by the authors. Licensee MDPI, Basel, Switzerland. This article is an open access article distributed under the terms and conditions of the Creative Commons Attribution (CC BY) license (http://creativecommons.org/licenses/by/4.0/). 